\section{Conhecimento, crença e \\ uso de medicina alternativa \\ e complementar por \\ fonoaudiólogas}

\author{
Knowledge, beliefs and \\ practices toward alternative and \\ complementary therapies among \\ speech-language pathologists
}

\section{Thaise Manzini'}

Edson Zangiacomi Martinez ${ }^{2}$

\section{Antonio Carlos Duarte de Carvalho²}

${ }^{1}$ Curso de Graduação em Fonoaudiologia, Faculdade de Medicina de Ribeirão Preto, USP Ribeirão Preto.

${ }^{2}$ Departamento de Medicina Social, Faculdade de Medicina de Ribeirão Preto, USP Ribeirão Preto.

Apoio financeiro: FUPEME - Fundação de Pesquisas Médicas de Ribeirão Preto.

Correspondência: Edson Zangiacomi Martinez. Departamento de Medicina Social, Faculdade de Medicina de Ribeirão Preto, Universidade de São Paulo (USP), Av. Bandeirantes 3900, CEP 14049 -

900 Ribeirão Preto, SP. E-mail: edson@fmrp.usp.br

\section{Resumo}

O objetivo do presente estudo é entender o conhecimento, as crenças e o uso das medicinas alternativas e complementares entre fonoaudiólogas que atuam no setor público de saúde. O estudo foi realizado em um hospital terciário de Ribeirão Preto, SP. Um questionário desenvolvido para os propósitos do estudo foi administrado em uma casuística de 40 fonoaudiólogas. Os itens do questionário foram criados conforme uma revisão da literatura. A aceitação de medicinas alternativas e complementares, como acupuntura e homeopatia, é expressiva entre as profissionais entrevistadas. Os resultados do presente estudo sugerem que o uso e a prática destas medicinas são feitos de forma racional, com certa prudência. As entrevistadas admitem que um tratamento complementar deve ser submetido a testes científicos antes de sua aceitação, e que é desaconselhável a um paciente buscar um tratamento alternativo ou complementar sem antes consultar o médico.

Palavras-chave: Medicinas alternativas e complementares. Fonoaudiologia. Conhecimento. Crenças. 


\section{Abstract}

The purpose of this study is to understand the knowledge, beliefs, concerns and practices toward alternative and complementary therapies among speech-language pathologists in a Brazilian public hospital. The current study was conducted in a large tertiary hospital in Ribeirão Preto, Southeastern Brazil. A questionnaire developed to measure knowledge, beliefs and practices on alternative and complementary therapies was answered by forty speech-language pathologists. The questionnaire items were created via literature review. Alternative and complementary therapies as acupuncture and homeopathy have high acceptance among the interviewed professionals. The results of the present study suggest that the use and practice of these techniques are made in a rational manner, with some prudence. The interviewed professionals admit that complementary treatment must be submitted to scientific tests before its acceptance, and that it is inadvisable for the patient to try treatment with alternative and complementary medicine before consulting a doctor.

Keywords: Alternative and complementary therapies. Speech-language pathology. Knowledge. Beliefs.

\section{Introdução}

Em 4 de maio de 2006 foi publicada no Diário Oficial da União a portaria de número 971 do Ministério da Saúde ${ }^{1}$, que criou a Política Nacional de Práticas Integrativas e Complementares no Sistema Único de Saúde (SUS). Esta portaria autorizou a utilização da homeopatia, acupuntura, fitoterapia e do termalismo social ou crenoterapia (uso de águas minerais) nos tratamentos do SUS, e recomendou a implantação e implementação das ações e serviços relativos às práticas integrativas e complementares pelas Secretarias de Saúde de Estados, Distrito Federal e Municípios. Logo após sua publicação, a Portaria 971 foi bastante criticada pelos profissionais da área médica por não estabelecer que diagnósticos e prescrições fossem competências exclusivas de profissionais formados em Medicina. As palavras de José Luiz Gomes do Amaral, então presidente da Associação Médica Brasileira (AMB), sumarizam estas críticas: "Entre a série de falhas graves, abre a possibilidade de que a acupuntura e a homeopatia sejam praticadas por profissionais de saúde diversos (ou multiprofissionais) inseridos no SUS, sem estabelecer claramente que o diagnóstico deve ser, obrigatoriamente, ato privativo dos médicos. Ela também tenta legitimar alternativas sem qualquer comprovação científica".

Ainda que uma parte da comunidade médica aponte equívocos no texto da Portaria 971, seguindo a mesma lógica dos acalorados debates em torno do Ato Médico, que colocaram em campos opostos médicos e outros profissionais da saúde, a adoção desta portaria no Sistema Público de Saúde pode contribuir intensamente para a popularização ainda maior das chamadas Medicinas Alternativas e Complementares (MAC) entre todas as camadas sociais atendidas pelo SUS.

A própria definição do que seriam as MAC é controversa. Em autores diferentes, ela aparece com diferentes sentidos e significados ${ }^{3}$. Para efeito deste trabalho adotaremos a definição proposta pela Organização Mundial de Saúde (OMS) sobre Medicina 
Tradicional ${ }^{4}$, para quem, em países como o Brasil, onde o sistema sanitário dominante se baseia na medicina alopática e onde as Medicinas Alternativas não se incorporaram no sistema sanitário nacional, elas são classificadas como MAC.

Portanto, segundo a OMS, MAC seria um conceito amplo, utilizado para referir-se tanto aos sistemas de Medicina Tradicional, como à medicina chinesa, à medicina ayurveda hindu e à medicina unani árabe, quanto às diversas formas de medicina indígena. As práticas da MAC incluiriam terapias com medicação, com uso de ervas, partes de animais e/ou minerais, e terapias sem medicação, como é o caso da acupuntura, das terapias manuais e das terapias espirituais. As práticas terapêuticas classificadas como alternativas ou complementares em geral não consideram a doença como resultante da intrusão de um agente externo, mas como um conjunto de causas que culminam em desarmonia e desequilíbrio ${ }^{5}$.

Embora existam muitas divergências sobre o que define uma medicina como complementar ${ }^{6}$, usaremos este termo de uma forma abrangente, sem questionar se o uso da medicina em questão é feito de forma concorrente ou paralela ao uso da medicina convencional, ou se as administrações são feitas ou não por um mesmo profissional. Usaremos o termo complementar para nos referirmos a estas medicinas quando usadas em união às práticas profissionais já estabelecidas. Finalmente, chamaremos de convencional aquela medicina aceita, praticada e ensinada nas escolas médicas, em oposição às medicinas alternativas e complementares, muito embora alguns autores $^{7}$ discordem do uso da expressão por considerarem que existe uma única medicina, não havendo, assim, a necessidade de rotulá-la como convencional.

Baseados nas reflexões de Furnham ${ }^{8}$, entendemos que é possível identificar cinco pontos que nos permitem diferenciar uma MAC da medicina convencional. O primeiro ponto se refere ao próprio conceito de saúde, que a medicina convencional define como ausência de doença, enquanto as MAC se referem a "desequilíbrios entre forças que se opõem” para caracterizar ou explicar os estados de saúde. O segundo ponto diz respeito ao conceito de doença, que é freqüentemente entendida pelos praticantes das MAC como uma desorganização entre forças energéticas que trazem, por conseqüência, sinais expressos pelo corpo, e não como um desvio localmente definido em um órgão ou tecido, como entende a medicina convencional. O terceiro ponto mostra diferenças entre os métodos de diagnóstico, sendo que os praticantes das MAC tendem a valorizar aspectos funcionais do indivíduo. As técnicas terapêuticas, onde se busca uma "revitalização energética", constituem o quarto aspecto que diferencia as MAC da medicina convencional. Neste contexto, os praticantes das MAC tendem a hostilizar os tratamentos químicos e cirúrgicos, comuns na medicina convencional. Finalmente, o quinto ponto é relacionado ao próprio paciente, que é incentivado pelos praticantes das MAC a ter uma participação mais ativa no processo terapêutico, sem se colocar no papel de um recipiente de soluções externas, como normalmente ocorre quando tratado pela medicina convencional.

Ainda neste esforço de buscar pontos que possibilitem uma clara diferenciação entre as chamadas MAC e a medicina dita convencional, fazemos nossas a reflexões de Luz ${ }^{9}$, para quem as MAC apresentam os seguintes aspectos que as diferenciam: primeiro, a reposição do sujeito doente como centro do paradigma médico; segundo, a re-situação da relação médico-paciente como elemento fundamental da terapêutica; terceiro, a busca de meios terapêuticos simples, despojados tecnologicamente, menos caros e com igual ou maior eficácia em termos curativos do que os meios comumente empregados pela medicina convencional; quarto, a construção de uma medicina que busca acentuar a autonomia do paciente e não sua dependência, como é comum na medicina convencional; e quinto, a afirmação de uma medicina que tem como paradigma central a categoria de saúde e não a de doença. 
Alheia à falta de comprovação científica de muitas MAC e às discussões entre médicos e outros profissionais da saúde sobre quais categorias são aptas para as utilizarem, uma grande parcela da população de muitos países vêm aderindo a estas formas de tratamento. Segundo Astin et al. ${ }^{10}$, entre $30 \%$ e $50 \%$ da população adulta de países industrializados utilizam alguma forma de MAC para prevenir ou tratar algum problema relacionado à saúde. Em uma pesquisa realizada entre os anos de 1997 e 2002 nos Estados Unidos ${ }^{11}$, estima-se que $35 \%$ da população adulta (cerca de 72 milhões de pessoas) deste país utilizam alguma MAC. Seus autores citam que a MAC mais usada pela população adulta dos Estados Unidos é a Fitoterapia $(18,6 \%$, representando mais de 38 milhões de americanos) seguida pelas técnicas de relaxamento, como massagens (14,2\% ou 29 milhões de indivíduos), e a quiroprática ( $7,4 \%$ ou 15 milhões de indivíduos). Em outro estudo também realizado nos Estados Unidos ${ }^{12}$, entre 1990 e 1997, estimou-se um aumento de $47,3 \%$ no número de visitas a terapeutas que utilizam as MAC, passando de 427 milhões em 1990 para 629 milhões em 1997. A partir deste estudo, verificouse também, no mesmo período (1990 a 1997), um aumento de $45,2 \%$ nos gastos da população com os serviços profissionais destes terapeutas, sendo estimado que os norte-americanos gastaram 21,2 bilhões de dólares em 1997, dos quais pelo menos 12,2 bilhões de dólares, pagos do próprio bolso. Os autores estimam que este valor é superior ao total gasto, no mesmo ano, em todas as hospitalizações pagas do próprio bolso.

Segundo Astin ${ }^{13}$, três fatores estão hipoteticamente relacionados à busca do paciente pelas MAC. O primeiro fator está associado à insatisfação com os tratamentos convencionais, motivada pela descrença em sua efetividade, pelos efeitos adversos produzidos, por julgá-los impessoais, ou por seu relativo alto custo. $\mathrm{O}$ segundo fator está ligado à crença de que as MAC trazem maior poder de escolha sobre as decisões a serem tomadas no decorrer do tratamento. E o terceiro fator diz respeito à suposta compatibilidade entre o princípio da MAC buscada e as crenças religiosas e espirituais do paciente, seus valores, seu modo de ver o mundo, e o seu próprio conceito de saúde e de doença.

Outros dados sugerem que um grande número de profissionais da saúde tem demonstrado algum interesse nas MAC. Em um estudo sobre as opiniões e conhecimentos de estudantes de medicina norte-americanos sobre MAC, Chez et al. ${ }^{14}$ observaram que grande parte dos entrevistados acreditava na utilidade clínica da acupuntura, da quiroprática, da medicina herbal, da meditação e da massagem. Por outro lado, poucos acreditavam na utilidade da homeopatia. Ao estudar as práticas e as crenças de médicos praticantes da medicina convencional em relação às MAC, Astin et $\mathrm{al}^{10}$ observaram que a metade dos entrevistados declarou acreditar na eficácia da acupuntura, da quiroprática (técnica que busca corrigir com as mãos problemas das articulações, dos músculos e, em especial, da coluna) e da massagem, enquanto um quarto acreditava na importância da homeopatia e $13 \%$ aprovavam as medicinas herbais. Lewith et al. ${ }^{15}$ enviaram questionários pelo serviço postal aos membros do Royal College of Physicians, Reino Unido, objetivando conhecer as atitudes e os conhecimentos dos médicos em relação MAC. Embora a metade dos 2.748 respondentes concorde fortemente com a afirmação de que estes métodos devam ser submetidos a mais testes científicos antes de serem aceitos pela medicina convencional, muitos aceitam métodos como a acupuntura, aromaterapia e massagem.

Estes e muitos outros estudos ${ }^{8,16-19}$ vêm demonstrando que, em diversas sociedades, a busca por MAC tem crescido de modo significativo nas últimas décadas.

Perante a crescente adesão de usuários de serviços de saúde às MAC, existe a necessidade de novos estudos sobre o conhecimento, atitudes e práticas dos diversos profissionais da saúde em relação a estas terapias. Estes estudos podem diagnosticar a adesão dos profissionais às medicinas não 
convencionais e as condutas em relação a elas, contribuindo para futuras discussões e revisões das políticas de práticas integrativas e complementares no SUS. Desta forma, o objetivo do presente estudo é avaliar o conhecimento, a crença e o uso das MAC por fonoaudiólogos que atuam em um hospital público de Ribeirão Preto, através de um questionário pré-estabelecido.

\section{Sujeitos e métodos}

Foram entrevistadas 40 fonoaudiólogas de três diferentes categorias funcionais: contratadas (14 profissionais, ou 35\%), aprimorandas (6, ou $15 \%)$ e aperfeiçoandas (20, ou $50 \%$ ), todas atuantes no Hospital das Clínicas da Faculdade de Medicina de Ribeirão Preto, da Universidade de São Paulo (USP). $\mathrm{O}$ aperfeiçoamento é um treinamento em serviço, com duração de um ano, em uma área específica da Fonoaudiologia. Houve boa receptividade entre as participantes convidadas a participar da pesquisa, havendo apenas duas recusas. O projeto de pesquisa e seu Termo de Consentimento Livre e Esclarecido foram previamente avaliados e aprovados pelo Comitê de Ética em Pesquisa (CEP) do Hospital das Clínicas da Faculdade de Medicina de Ribeirão Preto, de acordo com o Processo HCRP número 14978/2005. O Termo de Consentimento Livre e Esclarecido foi assinado por cada participante antes da entrevista.

O questionário usado para as entrevistas incluiu questões abertas sobre a idade, o ano de formatura e o tempo de atuação na Fonoaudiologia. Os 12 itens associados ao conhecimento e à prática de tratamentos alternativos e complementares abordados no questionário estão mostrados no Quadro 1. As fonoaudiólogas foram convidadas a responder a estes itens em uma escala de Likert de 8 pontos, variando de 0 a 7 , onde quanto maior a sua pontuação, maior seu grau de concordância em relação ao item. Estes itens foram baseados naqueles constantes dos instrumentos HCAMQ (Holistic Complementary and Alternative Medicine Questionnaire), desenvolvido por Hyland et al. ${ }^{20}$, CHBQ (CAM Health Belief Questionnaire), proposto por Lie e Boker ${ }^{21}$ e CAMBI (Complementary and Alternative Medicine Beliefs Inventory), proposto por Bishop et al. ${ }^{22}$. Foram escolhidos dentre os itens presentes nestes instrumentos aqueles que apresentavam maior compatibilidade com os propósitos do presente estudo, posteriormente adaptados às questões pertinentes à prática da Fonoaudiologia.

O questionário contém ainda questões sobre o conhecimento, crença e uso de uma série de MAC, listadas na Tabela 1. A escolha destas MAC foi motivada por suas citações em diversos estudos ${ }^{10,14,15,23}$. Na entrevista, um cartão contendo os nomes destas medicinas, em ordem aleatória, era entregue às fonoaudiólogas, sendo estas convidadas a responder a cinco questões: (a) quais destas MAC você conhece?; (b) em quais destas MAC você acredita?; (c) quais destas MAC você utiliza para seu tratamento pessoal?; (d) quais destas MAC você utiliza no tratamento de seus pacientes?; (e) e quais destas MAC você recomenda aos seus pacientes buscarem com outros especialistas? O uso destas MAC no tratamento de pacientes não necessariamente ocorre nos atendimentos realizados na instituição onde a pesquisa foi conduzida.

$\mathrm{Na}$ análise das respostas aos 12 itens associados ao conhecimento e à prática de tratamentos alternativos e complementares, utilizou-se a análise de correspondências (AC). Trata-se de uma técnica estatística multivariada apropriada à análise de dados em escala de Likert ${ }^{24,25}$, que permite medir e visualizar o grau de associação entre um conjunto de categorias de variáveis qualitativas. Quando aplicada aos dados do questionário de conhecimento e à prática de tratamentos alternativos e complementares, indica, de modo geral, a qual grau de concordância (na escala de 0 a 7 ) estão associados os diferentes itens, em um gráfico cartesiano denominado mapa de correspondências. Na AC, a variação total dos dados é denominada inércia, sendo esta variação decomposta em cada eixo (ou dimensão) do gráfico. Desta forma, a inércia associada a 
Quadro 1 - Itens associados ao conhecimento e à prática de tratamentos alternativos e complementares abordados no questionário, e seus rótulos utilizados na análise de correspondência.

Chart I -Items associated to the knowledge and practice of alternative and complementary treatments approached in the questionnaire and the labels used in the correspondence analysis

\begin{tabular}{|l|l|}
\hline Rótulo & Item \\
\hline A & $\begin{array}{l}\text { Um tratamento complementar deve ser submetido a testes científicos antes de ser } \\
\text { aceito pelo Conselho de Fonoaudiologia. }\end{array}$ \\
\hline B & $\begin{array}{l}\text { Tratamentos alternativos e complementares que não foram cientificamente } \\
\text { testados devem ser desencorajados. }\end{array}$ \\
\hline C & $\begin{array}{l}\text { Você previne seus pacientes de que pode ser perigoso fazer seu próprio } \\
\text { tratamento utilizando técnicas complementares ou alternativas. }\end{array}$ \\
\hline D & $\begin{array}{l}\text { A medicina alternativa só deve ser utilizada quando a medicina convencional não } \\
\text { tiver mais nada a oferecer. }\end{array}$ \\
\hline E & Tratamentos de saúde só devem utilizar ingredientes naturais. \\
\hline F & Eu prefiro remédios naturais aos medicamentos convencionais. \\
\hline G & $\begin{array}{l}\text { Um tratamento deve ser um complemento à minha habilidade natural de estar } \\
\text { saudável. }\end{array}$ \\
\hline H & Eu acredito que o meu corpo tem uma habilidade natural de se "curar". \\
\hline I & Os tratamentos devem fazer uso da tecnologia científica moderna. \\
\hline J & Os efeitos das terapias alternativas são o resultado do chamado "efeito placebo". \\
\hline K & $\begin{array}{l}\text { A maioria das terapias complementares e alternativas estimula o poder terapêutico } \\
\text { natural do corpo. }\end{array}$ \\
\hline L & $\begin{array}{l}\text { Não é aconselhável tentar um tratamento com a medicina alternativa e } \\
\text { complementar, antes de procurar um médico. }\end{array}$ \\
\hline
\end{tabular}

cada dimensão do gráfico nos informa qual é a proporção da variação total que aquele eixo está explicando.

A tabulação e a análise dos dados foram feitas através do programa de computador SAS versão 8 .

Os autores, através de documentação assinada e enviada a RBE, declaram não existir nenhum tipo de conflito de interesses.

\section{Resultados}

As 40 fonoaudiólogas entrevistadas, todas de sexo feminino, têm idades entre $22 \mathrm{e}$ 35 anos (em média, 25,9 anos, com um desvio padrão [DP] de 4,23 anos). Dentre estas 40 profissionais, 20 são aperfeiçoandas, com idades entre 22 e 26 anos (média 23,3, DP 1,41 ano), 6 são aprimorandas, com idades entre 22 e 24 anos (média 22,7, DP 0,82 ano) e 14 são fonoaudiólogas contratadas pela insti- tuição, com idades entre 25 e 35 anos (média 30,5, DP 3,35 anos). O tempo de experiência profissional varia de 1 mês a 14 anos, sendo que as aperfeiçoandas possuem de 1 mês a 5 anos de experiência (média 13,5 meses, DP 14,2 meses), as aprimorandas, de 1 mês a 1 ano (média 6,5 meses, DP 6,0 meses) e as fonoaudiólogas contratadas, de 2 a 14 anos (média 8,4 anos, DP 3,8 anos).

A Figura 1 mostra o mapa de correspondências gerado da análise das respostas aos 12 itens associados ao conhecimento, crença e uso de medicina alternativa e complementar, onde se verifica que os rótulos de A a L relativos aos itens (Quadro 1) e de 0 a 7 associados às respostas aos itens aparecem dispostos em uma curva em forma de parábola. Este padrão, comum em mapas de correspondência onde são analisados dados em escala de Likert, recebe o nome de efeito ferradura (horseshoe effect, ver, por exemplo, 
Tabela 1 - Freqüências relativas de fonoaudiólogas segundo conhecimento, crença, uso e atitude de recomendar a sua busca, conforme cada uma das terapias alternativas e complementares abordadas.

Table 1 - Relative frequencies of speech therapists according to knowledge, belief, use and attitude to recommend search, as per each of the alternative and complementary therapies approached

\begin{tabular}{|c|c|c|c|c|c|}
\hline Terapia & $\begin{array}{l}\text { Conhece a } \\
\text { terapia } \\
(\%)\end{array}$ & $\begin{array}{l}\text { Acredita na } \\
\text { terapia } \\
(\%)\end{array}$ & $\begin{array}{c}\text { Usa para } \\
\text { tratamento pessoal } \\
(\%)\end{array}$ & $\begin{array}{c}\text { Usa no tratamento } \\
\text { dos pacientes } \\
(\%)\end{array}$ & $\begin{array}{c}\text { Recomenda aos } \\
\text { pacientes buscarem } \\
(\%)\end{array}$ \\
\hline Acupuntura & 95,0 & 67,5 & 15,0 & 2,5 & 25,0 \\
\hline Auraterapia & 17,5 & 2,5 & & & \\
\hline Auriculoterapia & 27,5 & 12,5 & 5,0 & & 2,5 \\
\hline Cromoterapia & 70,0 & 12,5 & & & \\
\hline Cura pela mente & 32,5 & 7,5 & & & \\
\hline Do-In & 32,5 & 10,0 & 2,5 & & \\
\hline Ervas medicinais & 75,0 & 45,0 & 17,5 & 2,5 & 7,5 \\
\hline Essências florais & 67,5 & 27,5 & 12,5 & & 2,5 \\
\hline Fitoterapia & 42,5 & 17,5 & 12,5 & & 5,0 \\
\hline Florais de Bach & 52,5 & 25,0 & 20,0 & & 5,0 \\
\hline Hidroterapia & 75,0 & 55,0 & 5,0 & & 12,5 \\
\hline Hipnose & 62,5 & 7,5 & 2,5 & & \\
\hline Homeopatia & 85,0 & 62,5 & 37,5 & 5,0 & 15,0 \\
\hline Yoga & 80,0 & 40,0 & 5,0 & & 17,5 \\
\hline Massagens & 87,5 & 55,0 & 20,0 & 10,0 & 20,0 \\
\hline $\begin{array}{l}\text { Medicina } \\
\text { Antroposófica }\end{array}$ & 12,5 & 2,5 & 2,5 & & 2,5 \\
\hline Medicina Indiana & 7,5 & & & & \\
\hline $\begin{array}{l}\text { Medicina } \\
\text { Ortomolecular }\end{array}$ & 50,0 & 27,5 & 2,5 & & \\
\hline Medicina Psiônica & 2,5 & & & & \\
\hline Meditação & 55,0 & 20,0 & & & \\
\hline Musicoterapia & 90,0 & 45,0 & 2,5 & & 30,0 \\
\hline Naturopatia & 7,5 & & & & \\
\hline Quiroprática & 10,0 & & & & \\
\hline Reflexologia & 20,0 & 12,5 & 2,5 & & 2,5 \\
\hline Reiki & 52,5 & 15,0 & 7,5 & & 2,5 \\
\hline Shiatsu & 60,0 & 17,5 & 5,0 & & 7,5 \\
\hline Talassoterapia & 7,5 & & & & \\
\hline $\begin{array}{l}\text { Tratamento } \\
\text { Espiritual }\end{array}$ & 52,5 & 22,5 & 7,5 & & \\
\hline
\end{tabular}

Greenacre $^{25}$, p.127). Verifica-se neste mapa que as respostas 0 a 7 são representadas em ordem inversa quando projetadas na dimensão 1. Esta dimensão responde por $66,2 \%$ da inércia, ou seja, uma grande parte da variação dos dados é explicada por este eixo. Cada item do questionário é representado no mapa de correspondências por seu rótulo (de A a L, conforme o Quadro 1), sendo que os itens representados à esquerda do gráfico, próximos ao rótulo 7, associam-se a um maior grau de concordância, enquanto 


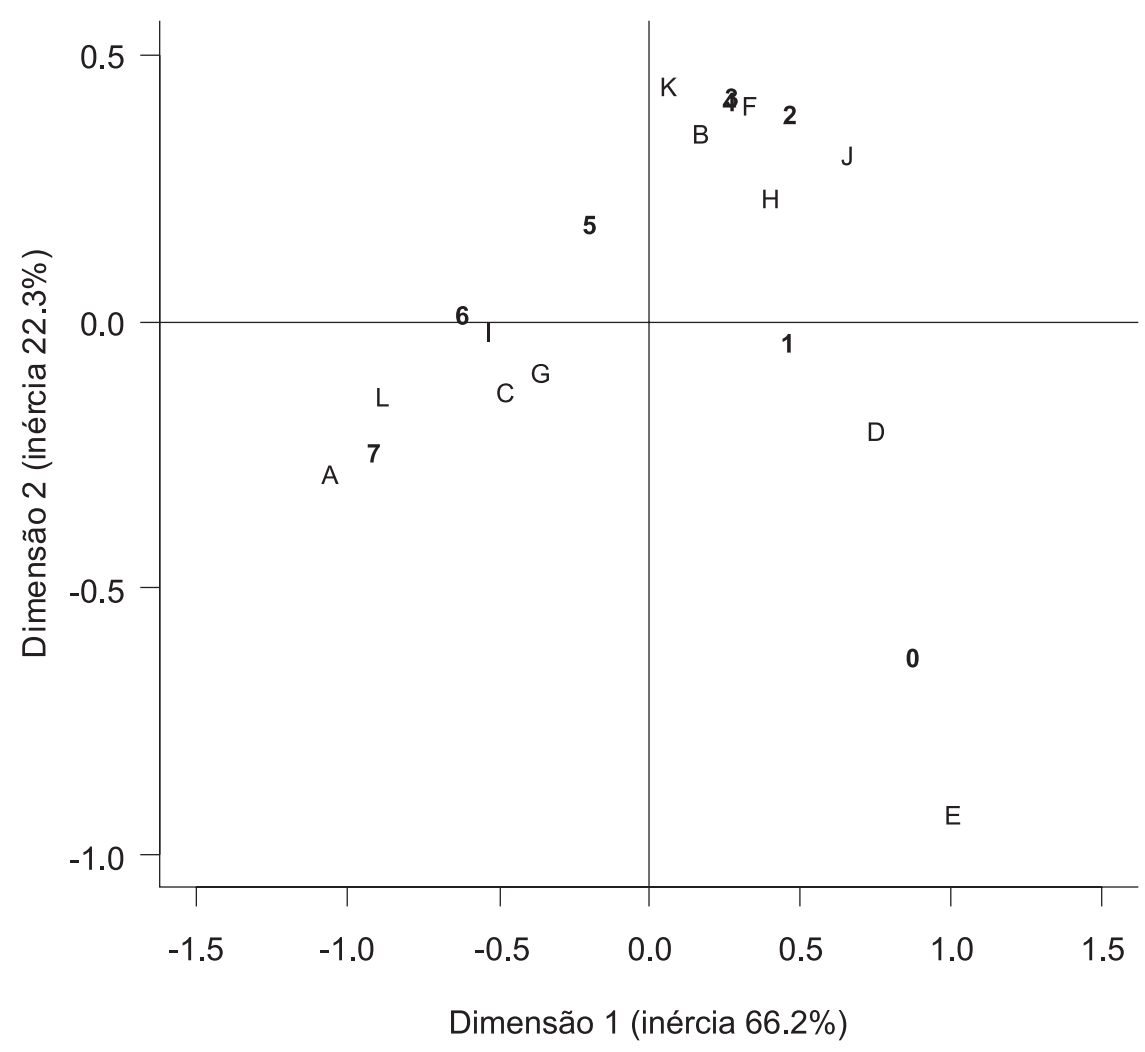

Figura 1 - Visualização das associações entre os itens abordados no questionário (de " $\mathrm{A}$ " a "L", ver Quadro 1) e o grau de concordância (de "0" a "7"), por intermédio do mapa gerado pela análise de correspondência.

Figure 1 - Visualization of the associations between the items approached in the questionnaire (from " $A$ " to " $L$ ", see Chart 1) and level of agreement (from " 0 " to " 7 "), by means of the map generated by the correspondence analysis.

os itens representados à direita, próximos ao rótulo 0 , relacionam-se a um menor grau de concordância.

Desta forma, a AC (Figura 1) evidencia que as fonoaudiólogas entrevistadas mostram prudência em relação às $\mathrm{MAC}$, ao concordarem com maior intensidade que um tratamento com tais medicinas deve ser submetido a testes científicos antes de ser aceito pelo Conselho de Fonoaudiologia, e que não é aconselhável tentar um tratamento com as MAC antes de procurar um médico (respectivamente, rótulos A e L, próximos ao rótulo 7 no mapa de correspondências, Figura 1). As participantes não concordam que os tratamentos de saúde só devam utilizar ingredientes naturais (rótulo $\mathrm{E}$, aquele representado mais próximo ao rótulo 0) e mostraram pouca concordância com a hipótese de que o corpo humano teria uma habilidade natural de cura (rótulo $\mathrm{H}$, próximo aos rótulos 1 e 2). Entendem ainda que os tratamentos devem fazer uso da tecnologia científica moderna, previnem seus pacientes de que pode ser perigoso fazer seu próprio tratamento utilizando medicinas complementares ou alternativas e concordam que um tratamento deve ser um complemento à habilidade natural de estar saudável (respectivamente, rótulos I, C e G, associados ao rótulo 6).

Por outro lado, mostram uma razoável aceitação em relação às MAC, ao não concordarem que elas só devem ser utilizadas quando a medicina convencional não tiver mais nada a oferecer (rótulo $\mathrm{D}$, próximo aos rótulos 0 e 1 quando projetados na dimensão 1) e que os efeitos das MAC seriam 
o resultado do chamado "efeito placebo" (rótulo J, associado ao rótulo 2).

As participantes expressaram alguma incerteza em relação ao possível estímulo que a maioria das MAC trariam ao poder terapêutico natural do corpo (rótulo K, próximo aos rótulos 3 e 4) e à afirmação de que tratamentos não cientificamente testados devem ser desencorajados (rótulo B).

A Tabela 1 mostra as freqüências relativas de conhecimento, crença, uso pessoal, uso para os pacientes e recomendação de cada uma das MAC listadas no questionário. Uma única entrevistada relatou não conhecer nenhuma destas medicinas e $68,4 \%$ afirmaram conhecer ao menos dez medicinas diferentes. Nota-se que a MAC mais conhecida entre as fonoaudiólogas é a acupuntura, conhecida por $95 \%(38 / 40)$ das entrevistadas, seguida da musicoterapia $(90 \%$, ou $36 / 40)$, massagens $(87,5 \%)$ e homeopatia $(85 \%)$.

Um total de $10 \%$ das entrevistadas (4/40) afirmou não acreditar em nenhuma destas medicinas listadas na Tabela 1, enquanto $20 \%$ dizem acreditar em ao menos dez e 42,5\% acreditam em, no mínimo, cinco. Observa-se que $62,5 \%$ das entrevistadas acreditam na homeopatia, sendo que $37,5 \%$ a utilizam para seu tratamento pessoal e $5 \%$ a utilizam no tratamento de seus pacientes. É também expressivo o número de fonoaudiólogas que acreditam nos benefícios das massagens, da hidroterapia, e das ervas medicinais. Dezessete entrevistadas (42,5\%) disseram não utilizar nenhuma destas técnicas para seu tratamento pessoal, enquanto uma mesma proporção (também 42,5\%) utiliza ao menos duas MAC dentre aquelas listadas na Tabela 1. As técnicas mais utilizadas pelas entrevistadas para seu tratamento pessoal são a homeopatia (37,5\%), as massagens (20\%), os florais de Bach (20\%), as ervas medicinais $(17,5 \%)$ e a acupuntura $(15 \%)$. Um número expressivo de profissionais recomenda aos seus pacientes buscarem a musicoterapia (30\%), a acupuntura (25\%) e as massagens $(20 \%)$.

Um resultado que se destaca é que somente $45 \%$ das entrevistadas acreditam na eficiência da musicoterapia, ainda que $90 \%$ relatem conhecer esta forma terapêutica. A rigor, a musicoterapia não é uma MAC, dado que algumas universidades públicas e particulares brasileiras já vêm oferecendo cursos de graduação específicos nesta área. Além disso, o projeto de lei que regulamenta a profissão de musicoterapeuta foi votado e aprovado em novembro de 2004 pela Câmara dos Deputados, em Brasília.

\section{Discussão}

Ao evidenciar que as fonoaudiólogas inseridas no setor público de saúde trazem atitudes positivas em relação às MAC, o presente estudo mostra alguma concordância com outros estudos realizados no Brasil, com outras categorias de profissionais da saúde. Por exemplo, ao procurar identificar as atitudes e as experiências com MAC entre os médicos domiciliados no Município de São Paulo, Akiyama ${ }^{26}$ evidenciou que $50 \%$ destes profissionais mostram atitude positiva com relação às MAC, $52 \%$ endossam ou prescrevem algum tipo, $20 \%$ referem treinamento e $13 \%$ informam ser provedores de pelo menos uma modalidade de MAC, sendo que 61,5\% acreditam que elas têm uma influência positiva no resultado terapêutico do paciente. Em uma outra pesquisa, Nuñes e Ciosak ${ }^{27}$ mostraram que $89 \%$ dos enfermeiros de um setor público de saúde de Santo Amaro, São Paulo, acreditam nas MAC e 11,1\% aplicam estas técnicas em seus pacientes.

Embora seja bastante expressiva a aceitação de MAC como a acupuntura e a homeopatia pelas fonoaudiólogas entrevistadas, os resultados do presente estudo sugerem que o uso e a prática destas medicinas são realizados de forma racional, acompanhados de certa prudência. Este comportamento se evidencia quando as entrevistadas admitem que um tratamento complementar deve ser submetido a testes científicos antes da sua aceitação pelo Conselho Federal de Fonoaudiologia, e que é desaconselhável a um paciente tentar um tratamento com a medicina alternativa $\mathrm{e}$ complementar antes de procurar um médi- 
co. As participantes mostraram uma visão responsável e cautelosa em relação a tais medicinas, ao admitirem que previnem seus pacientes de que pode ser perigoso fazer seu próprio tratamento utilizando MAC. Estas atitudes perante as MAC parecem estar em desacordo com os resultados obtidos por Ávila-Pires ${ }^{28}$, que postula que a escolha de um sistema terapêutico é mais influenciada por fatores culturais, pela tradição familiar e pela moda do que pela análise racional dos seus fundamentos.

A crescente busca da população pelas MAC, a expansão do seu oferecimento por profissionais da saúde em serviços públicos e particulares, e a recente proposta da Política Nacional de Práticas Integrativas e Complementares no SUS trazem não somente a necessidade de novos ensaios clínicos que busquem a comprovação ou não da eficiência e eficácia destes métodos terapêuticos, mas também de estudos sobre demandas, práticas, percepções, crenças e atitudes de profissionais de diferentes classes que integram o sistema de saúde. Estes estudos são importantes para a definição de novas políticas de saúde, auxiliando a discussão das regras para a inserção das práticas integrativas no SUS. O presente estudo trouxe informações sobre o conhecimento, a crença e o uso das MAC por fonoaudiólogas de um setor terciário de saúde. Novos estudos com base em uma população mais ampla de profissionais de Fonoaudiologia devem ser incentivados, de forma que a inserção destes profissionais nas políticas de práticas integrativas e complementares no SUS seja melhor direcionada.

\section{Referências}

1. Brasil. Ministério da Saúde. Portaria no 971, de 03 de maio de 2006. Aprova a Política Nacional de Práticas Integrativas e Complementares no SUS. Diário Oficial da União, Brasília, 2006. Disponível em: http://portal. saude.gov.br/portal/arquivos/pdf/portariafito.pdf. Acesso em 13 de maio de 2008.

2. Amaral JLG. Saúde não é moeda de negociação política (editorial). Jornal da Associação Médica Brasileira 2006; 47(1343): 3

3. Barros NF, Nunes ED. Medicina Alternativa e Complementar no Brasil: um conceito e diferentes significados. Cad Saúde Pública 2006, 22(10): 2023-8.

4. World Health Organization. National policy on traditional medicine and complementary/alternative medicine. Geneva: World Health Organization; 2002.

5. Queiroz MS. O itinerário rumo às medicinas alternativas: uma análise em representações sociais de profissionais da saúde. Cad Saúde Pública 2000; 16(2): 363-75.

6. Low J. Alternative, complementary or concurrent health care? A critical analysis of the use of the concept of complementary therapy. Complement Ther Med 2001; 9(2): 105-10.

7. Marusic M. "Complementary and alternative” medicine - a measure of crisis in academic medicine. Croat Med J 2004; 45(6): 684-8.

8. Furnham A. Complementary and alternative medicine. The Psychologist 2002; 15(5): 228-31.
9. Luz MT. Cultura contemporânea e medicinas alternativas: novos paradigmas de saúde no fim do século XX. PHYSIS: Rev Saúde Coletiva 2005; 15(supl.): 145-176.

10. Astin JA, Marie A, Pelletier KR, Hansen E, Haskell WL. A review of the incorporation of complementary and alternative medicine by mainstream physicians. Arch Intern Med 1998; 158(21): 2303-10.

11. Tindle HA, Davis RB, Phillips RS, Eisenberg DM. Trends in use of complementary and alternative medicine by US adults: 1997-2002. Altern Ther Health Med 2005; 11(1): 42-9.

12. Eisenberg DM, Davis RB, Ettner SL, Appel S, Wilkey S, Van Rompay M, Kessler RC. Trends in alternative medicine use in the United States, 1990-1997: results of a follow-up national survey. JAMA 1998; 280(18): 1569-75.

13. Astin JA. Why patients use alternative medicine: results of a national study. JAMA 1998; 279(19): 1548-53.

14. Chez RA, Jonas WB, Crawford C. A survey of medical students' opinions about complementary and alternative medicine. Am J Obstet Gynecol 2001; 185(3): 754-7.

15. Lewith GT, Hyland M, Gray SF. Attitudes to and use of complementary medicine among physicians in the United Kingdom. Complement Ther Med 2001; 9(3): 167-72.

16. Fisher P, Ward A. Complementary medicine in Europe. Br Med J 1994, 309(6947): 107-11. 
17. White A, Ernst E. Economic analysis of complementary medicine: A systematic review. Complement Ther Med 2000, 8(2): 111-18.

18. Ernst E, Furnham A. BMWs and complementary/ alternative medicine. Focus on Alternative and Complementary Therapies 2000, 5(4): 253-4.

19. Barnes PM, Powell-Griner E, McFann K, Nahin RL. Complementary and alternative medicine use among adults: United States, 2002. Advance Data From Vital and Health Statistics 2004, 343: 1-20.

20. Hyland ME, Lewith GT, Westoby C. Developing a measure of attitudes: the holistic complementary and alternative medicine questionnaire. Complement Ther Med 2003, 11(1), 33-8.

21. Lie D, Boker J. Development and validation of the CAM Health Belief Questionnaire (CHBQ) and CAM use and attitudes amongst medical students. BMC Medical Education 2004, 4: 2.

22. Bishop FL, Yardley L, Lewith G. Developing a measure of treatment beliefs: The complementary and alternative medicine beliefs inventory. Complement Ther Med 2005, 13(2): 144-9.

23. Greenfield SM, Innes MA, Allan TF, Wearn AM. First year medical students' perceptions and use of complementary and alternative medicine. Complement Ther Med 2002, 10(1): 27-32.
24. Colugnati FAB. Uma alternativa para análise de instrumentos psicométricos baseados em escala de Likert via Análise de Correspondência Múltipla [dissertação de mestrado]. Campinas: Instituto de Matemática, Estatística e Computação Científica da Universidade Estadual de Campinas; 2000.

25. Greenacre MJ. Correspondence analysis in practice. London: Academic Press; 1993.

26. Akiyama K. Práticas não-convencionais em medicina no Município de São Paulo [tese de doutorado]. São Paulo: Faculdade de Medicina da Universidade de São Paulo, Departamento de Medicina Preventiva, Universidade de São Paulo; 2004.

27. Nuñes HMF, Ciosak SI. Terapias alternativas/ complementares: o saber e o fazer das enfermeiras do distrito administrativo 71 - Santo Amaro - São Paulo. Rev Esc Enferm USP 2003; 37(3): 11-8.

28. Ávila-Pires FD. Ponto de vista: teoria e prática das práticas alternativas. Rev Saúde Publica 1995; 29(2): 147-51.

Recebido em: 04/12/06 Versão final reapresentada em: 21/09/07 Aprovado em: 18/02/07 